\title{
Structural studies of the Tudor domain from the Bombyx homolog of Drosophila PAPI: Implication to piRNA biogenesis
}

Paul A. Hubbard ${ }^{1 \pi}$, Xinlei Pan ${ }^{1 \pi}$, Randall McNally ${ }^{1}$, Yohei Kirino ${ }^{2}$ and Ramachandran Murali ${ }^{1 *}$

${ }^{1}$ Department of Biomedical Sciences, Research Division of Immunology, Cedars-Sinai Medical Center, Los Angeles, CA, USA

${ }^{2}$ Computational Medicine Center, Sidney Kimmel Medical College, Thomas Jefferson University, Philadelphia, PA, USA

${ }^{*}$ Corresponding author

E-mail: Ramachandran.Murali@csmc.edu

"These authors contributed equally to this work. 


\section{ABSTRACT}

PIWI proteins and their associated PIWI-interacting RNAs (piRNAs) play crucial roles in proper gametogenesis in animal gonads. Partner of PIWls (PAPI) is one of the important piRNA biogenesis factors. PAPI contains a Tudor domain and tandem $\mathrm{KH}$ domains. The tudor domain specifically recognizes symmetrical-dimethylarginines (sDMAs) on PIWI proteins. BmPAPI, a Bombyx mori homolog of PAPI, is localized at the outer membrane of mitochondria and supports exonucleolytic trimming of piRNA precursors to form mature 3'-end of piRNAs. To understand the structural basis of piRNA processing by BmPAPI, we present crystal structures of the apo- and sDMA-liganded Tudor domain of BmPAPI.

\section{INTRODUCTION}

PIWI proteins are a subfamily of Argonaute (Ago) proteins, which form gene-silencing complexes with PIWl-interacting RNAs (piRNAs). They play important roles in germline development and are well-conserved through evolution (1-4). The PIWI-piRNA machinery protects genomic integrity in animal gonads by silencing transposons, and regulates gene expression to ensure proper gametogenesis (5-7). Disruption of piRNA biogenesis causes germline defects and eventually leads to sterility $(8,9)$.

PIWI proteins contain four main domains; an N domain, a PAZ domain that recognizes the 3' end of the interacting RNA, a MID domain that coordinates the 5' phosphate of the RNA, and a PIWI domain that houses key catalytic residues $(10,11)$. In addition, symmetricaldimethylarginines (sDMAs) are conserved in PIWI proteins from various organisms and are present in motifs that contain multiple arginine-glycine or arginine-alanine repeats, typically at the N-termini of PIWI proteins $(12,13)$. It is reported that arginine methylation is crucial for PIWImediated piRNA biogenesis $(14,15)$. 
The Tudor royal family of proteins recognize methylated lysine or arginine residues to mediate protein-protein interactions, and are characterized by the well-studied canonical Tudor core domain structure, which typically comprises 4-5 $\beta$-strands that form a $\beta$-barrel with an aromatic pocket on the surface to accommodate methylated substrate $(16,17)$. A subset of the Tudor domain-containing proteins, primarily expressed in the germ cells, binds PIWI proteins through specific interactions with sDMA and is essential in piRNA biogenesis (18-21). PIWI-binding Tudor proteins possess an 'extended Tudor domain' with an extra conserved structural element flanking the Tudor core $(22,23)$. Additionally, in these proteins, the Tudor domain is frequently associated with RNA-binding domains such as the hnRNP K Homology (KH) domain (24-26).

PAPI (Partner of PIWIs) is a Tudor domain-containing protein first discovered in Drosophila that specifically interacts with PIWI proteins through recognition of sDMA (27). It plays an essential role in piRNA biogenesis by recruiting PIWI proteins to nuage, the perinuclear granules in Drosophila germline cells. Mouse and Bombyx mori studies have revealed that disruption of PAPI causes 3'-end extension of piRNAs, suggesting its role in 3'-end maturation of piRNAs (28, 29). Indeed, a recent report showed that BmPAPI, a Bombyx mori homolog of Drosophila PAPI, supports PNLDC1-catalyzed processing of PIWI-loaded piRNA precursors to form mature 3'ends of piRNAs $(28,30)$. BmPAPI contains tandem $\mathrm{KH}$ domains and an extended Tudor domain consisting of a core Tudor domain flanking an oligonucleotide-binding fold (OB-fold) domain. The Tudor domain specifically recognizes sDMAs on Bombyx PIWI proteins, Siwi and BmAgo3, and drives PIWI-BmPAPI interaction at the outer membrane of mitochondria (28). While the $\mathrm{KH}$ domains are expected to interact with piRNAs and/or piRNA precursors, supporting evidence for this is needed and its function in the piRNA biogenesis pathway remains unclear. To better characterize BmPAPI function and the PIWI-PAPI interaction, we initiated the structural studies 
of BmPAPI. Here we report the crystal structures of the BmPAPI Tudor domain in its unliganded form as well as bound to sDMA. The overall topology of the Tudor domain and the binding site of sDMA is conserved in BmPAPI. An extended loop forms part of the sDMA-binding site which may have functional role in piRNA biogenesis.

\section{METHODS:}

\section{Expression and purification of BmPAPI Tudor domain}

The BmPAPI extended Tudor domain region corresponding to Gly245-Gly463 was sub-cloned into pET28a vector to include an N-terminal His-tag and expressed in BL21(DE3). Purification was by IMAC and SEC using a HiLoad 16/60 Superdex column (GE Lifesciences) to produce $>95 \%$ pure protein as determined by SDS-PAGE analysis.

\section{Pull-down binding assay}

To confirm the ability of the purified BmPAPI Tudor domain to recognize sDMAs of PIWI proteins, stable BmN4 cells expressing Flag-tagged Siwi proteins were utilized for pull-down binding assay as described previously (28). Briefly, the cells expressing wild-type Siwi or sDMAlacking mutant Siwi, whose sDMAs are replaced with lysines, were cultured at $27^{\circ} \mathrm{C}$ in InsectXpress medium (Lonza). Cell lysates were prepared in a binding buffer containing $50 \mathrm{mM} \mathrm{Na-}$ Phosphate (pH 8.0), $300 \mathrm{mM} \mathrm{NaCl}, 0.01 \%$ Tween 20, 1\% Triton X-100, and complete protease inhibitor cocktail (Roche Diagnostics). BmPAPI Tudor protein immobilized on Dynabeads HisTag Isolation and Pulldown (Life Technologies) was incubated with the lysate for 20 min at $4^{\circ} \mathrm{C}$ and was washed extensively with binding buffer. Bound proteins were then eluted into an elution buffer containing $50 \mathrm{mM}$ sodium phosphate, $\mathrm{pH} 8.0,300 \mathrm{mM}$ imidazole, $140 \mathrm{mM} \mathrm{NaCl}$ and $0.02 \%$ Tween 20 . Western blots of the proteins were subsequently performed by using anti-Flag (Sigma Aldrich) for detection of Siwi and anti-His (Cell Signaling) for detection of BmPAPI Tudor. 


\section{Crystal structures of BmPAPI Tudor domain}

Protein was concentrated to $10 \mathrm{mg} / \mathrm{mL}$ in $20 \mathrm{mM}$ HEPES, $\mathrm{pH} 7.5$ and $150 \mathrm{mM} \mathrm{NaCl}$ before being crystallized using the hanging drop method at $18^{\circ} \mathrm{C}$. For the apo-BmPAPI crystals, the well solution contained 1\% PEG 8,000, $50 \mathrm{mM}$ MES, pH 5.5, $100 \mathrm{mM} \mathrm{KCl}$ and $10 \mathrm{mM} \mathrm{MgCl}_{2}$, and was mixed with protein at an equal volume ratio, with crystals of space group $\mathrm{P} 3{ }_{2} 21$ taking about one week to reach their maximum dimensions of $90 \times 90 \times 30 \mu \mathrm{m}$. Prior to data collection, crystals were dehydrated against air in a sealed chamber for two days before being mounted on nylon loops and cooled to $100 \mathrm{~K}$. To solve the structure of the BmPAPI-sDMA complex, attempts to obtain apo-crystals from the previous conditions failed. Thus, a new form of apoBmPAPI crystals (space group $\mathrm{P}_{3}{ }_{2}{ }_{12}$ ) was obtained using the hanging drop method with a precipitant solution of $0.95 \mathrm{M}$ ammonium sulfate, $50 \mathrm{mM}$ sodium acetate, $\mathrm{pH} 5.5$ and $5 \%$ glucose. These crystals were untreated or soaked in $50 \mathrm{mM}$ sDMA salt (Sigma Aldrich) for 3 days before data collection. X-ray diffraction data were collected in-house using a Rigaku MicroMax 007-HF rotating anode equipped with a Rigaku R-AXIS IV++ image plate. BmPAPI structures were solved using the molecular replacement method. The $\mathrm{P} 3_{2} 21$ apo- form was determined using human Tudor domain (PDB ID: 3FDR) as the search model. Subsequently, the apo- and sDMA-forms of the $\mathrm{P} 4_{3} 2{ }_{1} 2$ crystals were determined using the apo-BmPAPI Tudor domain. Approximately $90 \%$ of residues were traced (excluding His-tag), with the models possessing appropriate R-work factors of $20.1 \%, 25.3 \%$, and $19.5 \%$ and R-free factors of $27.7 \%$, 29.6\%, and $26.5 \%$ for the $P 3_{2} 21$ apo- (PDB ID 5VQG), $P 4_{3} 2{ }_{1} 2$ apo- (PDB ID 5VY1), and $P 4_{3} 2{ }_{1} 2$ sdMA-bound (PDB ID 5VQH) forms, respectively.

\section{RESULTS AND DISCUSSION}




\section{Tudor domain of BmPAPI specifically binds to sDMAs of PIWI protein}

To characterize the Tudor domain of BmPAPI and to confirm its ability to specifically recognize sDMAs of PIWI proteins, we produced and purified His-tagged Tudor recombinant protein and performed pull-down experiments. We immobilized the recombinant Tudor on beads and subsequently incubated the beads with lysates from BmN4 stable cell lines expressing wild-type or mutant Siwi protein. The mutant Siwi protein lacks sDMAs due to substitutions of arginines in the sDMA motif with lysines (Fig. 1A). After extensive washing, the eluates were subjected to western blots to detect the bound Siwi protein. As shown in Fig. 1B, only wild-type Siwi, and not mutant Siwi, was detected in the Tudor-bound fraction, suggesting specific association of the BmPAPI Tudor domain with the sDMAs of Siwi protein.

\section{Crystal structure of the extended Tudor domain from BmPAPI}

To determine the structure of the extended Tudor domain of BmPAPI, crystallization conditions were screened and optimized to yield crystals of space group $P 3_{2} 21$ that diffracted to $2.6 \AA$ (Table 1). The refined apo-model contains one protein molecule and 56 water molecules in the asymmetric unit, showing electron density for residues 245-461, excluding flexible loop region residues 379-391, 424-429 and 444-445. The extended Tudor domain of BmPAPI consists of an OB-fold domain and a core Tudor domain (31). The secondary structure comprises 6 -helices and $10 \beta$-sheets, as is shown in Figure 2. A closed $\beta$-barrel is present in the OB-fold domain, comprising anti-parallel $\beta$-sheets $\beta 1-\beta 2$ and $\beta 7-\beta 9$, and capped by $\alpha$-helix $\alpha 5$ located between $\beta 7$ and $\beta 8$. It has been reported that the OB-fold domain of a Drosophila homolog protein was responsible for sensing the position of the sDMA (22). Whether the OB-fold domain of BmPAPI has a similar function is not yet known. The core Tudor domain is formed by four anti-parallel $\beta$ sheets, $\beta 3-\beta 6$. Two long linkers connect the core Tudor domain to the OB-fold domain: helices $\alpha 1-\alpha 2$ at the N-terminus of the core Tudor domain and $\alpha 3-\alpha 4$ at the C-terminus. 


\section{Crystal structure of the BmPAPI-sDMA complex}

To better understand the interaction between BmPAPI and PIWI proteins, we attempted to solve the structure of sDMA-bound BmPAPI Tudor domain by soaking the apo- crystals with sDMA ligand, but were unsuccessful. However, we found crystals of a different space group $\left(\mathrm{P}_{3}{ }_{2}{ }_{1} 2\right)$ that were amenable to soaking with sDMA ligand, and solved the structure of the $P 4_{3} 2{ }_{1} 2$ form in both apo- and sDMA-bound states (Table 1). The structures are solved to $3.05 \AA$ and $2.4 \AA$ (for the apo- and sDMA-bound forms, respectively) by molecular replacement using the apoBmPAPI Tudor domain as a search model. The refined sDMA-bound structure contains two protein chains, $A$ and $B$, and 153 water molecules in the asymmetric unit. Chain $A$ and Chain $B$ have a RMSD of $0.456 \AA$ for $177 \mathrm{C} \alpha$ atoms aligned (Figure $3 \mathrm{~A}$ ).

The overall structures of the $\mathrm{P}_{2} 21$ apo-BmPAPI and $\mathrm{P}_{3} 2_{1} 2$ sDMA-BmPAPI complex are highly similar; the RMSD for backbone structures of apo-BmPAPI and sDMA-BmPAPI (Chain B) is $0.610 \AA$ for $177 \mathrm{Ca}$ atoms aligned. However, comparison of the two structures revealed that the loop consisting of residues 308-314 and inserted between $\beta$-strands $\beta 3$ and $\beta 4$ has moved in the sDMA-bound form to enclose the sDMA inside the aromatic cage (Figure 3B). A closer view of the loop region shows that in the apo- state (open position), Tyr315 is double hydrogenbonded to Arg313 while His310 forms a hydrogen bond with Glu351 of helix a3 (Figure 3C). While two conformations of the sDMA binding pockect have been observed in the structure of SMN Tudor domain (32), it must be noted that the loop of $\mathrm{P}_{4}{ }_{2}{ }_{1} 2$ sDMA-BmPAPI is fashioned at the surface and interacting with crystal symmetry equivalents, raising the possibility that the observed conformational changes are influenced by crystal packing. Indeed, the loop in one of the two molecules in the asymmetric unit of the $\mathrm{P}_{4}{ }_{3}{ }_{1} 2$ apo-form is in the sDMA-bound conformation (the loop in the other molecule is partially disordered). Further studies, perhaps including structure determination of the full-length protein, are necessary to resolve whether sDMA binding influences the position of the loop or if the movement described here is merely a 
product of crystal packing.

\section{Comparison of BmPAPI and other Tudor domain-containing proteins}

Tudor domains are conserved through many eukaryotic species. A search using BLAST shows that PAPI protein from Drosophila melanogaster (accession number NP_608657.1) shares 45\% sequence identity with the extended Tudor domain of $\operatorname{BmPAPI}(33,34)$. The top hits with known structure from human, mouse and Drosophila melanogaster are human Tdrd2 (PDB ID: 5J39, chain A), extended Tudor domain Td3 from mouse Tdrd1 (PDB ID: 4B9W, chain A), and eTud11 domain of Drosophila melanogaster Tudor protein (PDB ID: 3NTI), sharing a sequence identity of $41 \%, 31 \%$ and $34 \%$ with BmPAPI, respectively. Figure S1 shows the alignment of these four Tudor proteins by the COBALT multiple alignment tool (35). While the structure of Drosophila PAPI remains unknown, the structures of the other three Tudor domains, chain A of human Tdrd2, chain A of mouse Tdrd1 TD3 domain and chain A of Drosophila Tudor eTud11 domain, align well with the sDMA-bound BmPAPI structure, with RMSD values of $1.2,1.9$ and $2.2 \AA$ for 153 residues aligned respectively, based on protein structure comparison service PDBeFold at European Bioinformatics Institute (Figure 4) (20, 22, 36-38).

\section{Aromatic cage of the core Tudor domain for sDMA binding}

Similar to other PIWI-binding Tudor domains, four key residues of the BmPAPI core Tudor domain constitute the aromatic cage for sDMA binding: Phe308 in the loop region between $\beta 3$ and $\beta 4$, Tyr315 on $\beta 4$, Tyr338 at the C-terminal end of $\beta 5$, and Tyr341 on the loop linking $\beta 5$ and $\beta 6$ (Figure $5 \mathrm{~A}$ ). The four aromatic residues coordinate the guanidino group of sDMA in four orientations, with Tyr338 in the back, Tyr315 on the bottom, and Tyr341 and Phe308 sandwiching sDMA on each side. The plane of the guanidino group is approximately parallel to the aromatic rings of Phe308 and Tyr341. Figure 5B shows the negatively-charged surface of the aromatic cage area, which favors the binding of the positively-charged guanidino group of 
sDMA. All four residues form cation-m interactions with one of the two methylated amine groups, at distances of $3.7 \AA, 4.1 \AA, 5.3 \AA$ and $3.7 \AA$ between the nitrogen atom and the center of the benzene rings. The other methylated amine group is hydrogen-bonded to Asp343 on $\beta 6$ to further enhance the binding interaction. A study on a homolog protein, Drosophila Tudor eTud11, identified a hydrogen bond between the amine group of sDMA and an asparagine residue in a similar position to BmPAPI Asp343 (22), and found that eliminating the hydrogen bond through mutagenesis caused a greater than 30 -fold reduction of sDMA binding affinity compared to wild-type Tudor, indicating the critical role of this interaction in SDMA recognition. Although mutagenesis and affinity assays are yet to be conducted to confirm the role of BmPAPI Asp343 in sDMA binding, we expect it to be significant based on the high similarity between BmPAPI and Drosophila eTud11.

\section{Surface potential of the extended Tudor domain of BmPAPI}

At the C-terminus, BmPAPI contains two highly charged $\mathrm{KH}$ RNA-binding domains. To understand whether there is any compensatory charge distribution at the sDMA-binding domain, which might act to stabilize the protein, we examined the surface potential of the BmPAPI Tudor. The extended Tudor domain has opposing surface potentials on the sides proximal and distal to the sDMA binding site (Figure $5 \mathrm{C}$ ). The surface of the side proximal to the sDMA binding site is mainly negatively charged (Figure 5D), whereas the majority of the surface on the distal side is positively charged, except for a few pockets. Considering that the N-terminus of BmPAPI contains a transmembrane domain preceding Tudor, we speculate that the surface distribution of Tudor might have a role in co-localization of BmPAPI and a PIWI protein on the outer surface of the mitochondria (28); the positively-charged distal side will favorably interact with anionic phospholipids of the membrane while the proximal side remains open for PIWI protein recruiting and docking. Validation for this role of the surface charge distribution is an ongoing investigation. 


\section{SUMMARY}

BmPAPI, a Bombyx homolog of the Drosophila PAPI protein, has been reported to play a crucial role in 3'-end formation of mature piRNAs. In this article we present the crystal structures of the extended Tudor domain of BmPAPI, both in its native state and in complex with sDMA. Since the crystals are in a different space group, the possibility of packing-induced conformational change could not be assessed. Nonetheless, a conformational change in the sDMA-binding site of a Tudor domain upon sDMA binding may be possible in the context of RNA binding to the two $\mathrm{KH}$ domains. In summary, the structural information herein reveals the molecular details of the interaction between the PAPI Tudor domain and SDMA, furthering understanding of the role of the PAPI-PIWI interaction in piRNA biogenesis pathways.

\section{AKNOWLEDGEMENT}

This work was supported by the National Institutes of Health grant (GM106047, to YK/RM) and a Japan Society for the Promotion of Science Postdoctoral Fellowship for Research Abroad (to SH). We would like to thank technical support provided by Drs. Akashi Otaki and Shozo Honda in preparation of bacterial expression system. 


\section{TABLE AND FIGURES}

Table 1. Data collection and refinement statistics.

\begin{tabular}{|c|c|c|c|}
\hline \multicolumn{4}{|l|}{ Data collection } \\
\hline & apo-BmPAPI Tudor & $\begin{array}{l}\text { sDMA-BmPAPI } \\
\text { Tudor }\end{array}$ & apo-BmPAPI Tudor \\
\hline $\begin{array}{l}\text { Resolution range } \\
(\AA)\end{array}$ & $\begin{array}{l}31.78-2.60(2.72- \\
2.60)^{\mathrm{a}}\end{array}$ & $\begin{array}{l}39.61-2.40(2.51- \\
2.40)\end{array}$ & $\begin{array}{l}19.50-3.05(3.20- \\
3.05)\end{array}$ \\
\hline Space group & $P 3_{2} 21$ & $\mathrm{P} 4{ }_{3} 2{ }_{1} 2$ & $\mathrm{P} 4_{3} 2_{1} 2$ \\
\hline $\begin{array}{l}\text { Unit cell dimensions } \\
a \times b \times c(\AA)\end{array}$ & $63.6 \times 63.6 \times 100.7$ & $66.0 \times 66.0 \times 224.5$ & $65.7 \times 65.7 \times 225.4$ \\
\hline $\begin{array}{l}\text { Unit cell angles } \alpha \times \\
\beta \times Y\left(^{\circ}\right)\end{array}$ & $90.0 \times 90.0 \times 120.0$ & $90.0 \times 90.0 \times 90.0$ & $90.0 \times 90.0 \times 90.0$ \\
\hline $\mathrm{R}_{\text {merge }}(\%)$ & $10.6(60.8)$ & $7.7(53.5)$ & $17.1(57.2)$ \\
\hline $\begin{array}{l}\text { Total number of } \\
\text { observed reflections }\end{array}$ & $31231(3665)$ & $68909(6867)$ & 26088 (8655) \\
\hline $\begin{array}{l}\text { Total number of } \\
\text { unique reflections / } \\
\text { multiplicity }\end{array}$ & $\begin{array}{l}7573(915) / 4.1 \\
(4.0)\end{array}$ & $\begin{array}{l}18600(2275) / 3.7 \\
(3.0)\end{array}$ & $\begin{array}{l}8655(1274) / 3.0 \\
(3.0)\end{array}$ \\
\hline Mean $1 / \sigma$ & $7.8(1.6)$ & $8.7(1.6)$ & $5.9(2.1)$ \\
\hline Completeness (\%) & $99.5(100.0)$ & $92.8(95.0)$ & $87.9(90.3)$ \\
\hline \multicolumn{4}{|l|}{ Refinement } \\
\hline $\mathrm{R}_{\text {work }} / \mathrm{R}_{\text {free }}(\%)$ & $20.1 / 27.7$ & $19.5 / 26.5$ & $25.3 / 29.6$ \\
\hline $\begin{array}{l}\text { r.m.s.d. bond } \\
\text { lengths }(\AA) / \text { angles } \\
\left({ }^{\circ}\right)\end{array}$ & $0.011 / 1.416$ & $0.012 / 1.482$ & $0.002 / 0.654$ \\
\hline $\begin{array}{l}\text { Number of residues } \\
\text { modeled }\end{array}$ & 195 (1 chain) & 398 (2 chains) & 389 (2 chains) \\
\hline $\begin{array}{l}\text { Number of water } \\
\text { molecules modeled }\end{array}$ & 56 & 153 & 0 \\
\hline $\begin{array}{l}\text { Average B-factor of } \\
\text { all residues }\left(\AA^{2}\right)\end{array}$ & 34.6 & 45.2 & 51.0 \\
\hline
\end{tabular}




\begin{tabular}{|c|l|l|l|}
\hline $\begin{array}{l}\text { Average B-factor of } \\
\text { all waters }\left(\AA^{2}\right)\end{array}$ & 25.2 & 40.1 & N/A \\
\hline $\begin{array}{l}\text { Ramachandran plot } \\
\text { (RAMPAGE): }\end{array}$ & \multicolumn{3}{|l|}{} \\
\hline Favored & $190(97.4 \%)$ & $378(95.0 \%)$ & $367(97.9 \%)$ \\
\hline Allowed & $5(2.6 \%)$ & $19(4.8 \%)$ & $8(2.1 \%)$ \\
\hline Outliers & $0(0.0 \%)$ & $1(0.3 \%)$ & $0(0 \%)$ \\
\hline
\end{tabular}

${ }^{a}$ Values in parentheses are for highest-resolution shell

Figure 1

A



B
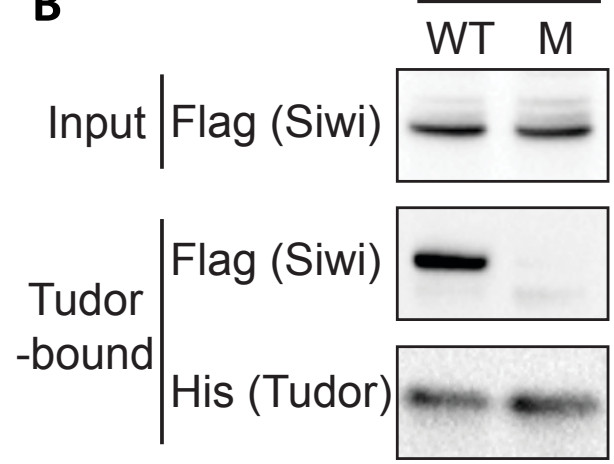

Figure 1. Pull-down experiments to analyze the association of BmPAPI Tudor domain with Siwi protein. (A) Domain structure of Siwi wild-type (WT) and mutant (M) Siwi protein sequences showing the arginines in the sDMA motif and their substitution by lysines in the mutant. (B) HisTudor immobilized on Dynabeads was incubated with the lysates from BmN4 cells expressing WT or M Siwi protein. After extensive washing, the eluates were probed with anti-Flag (for Siwi detection) and anti-His antibodies (for Tudor detection). 
Figure 2

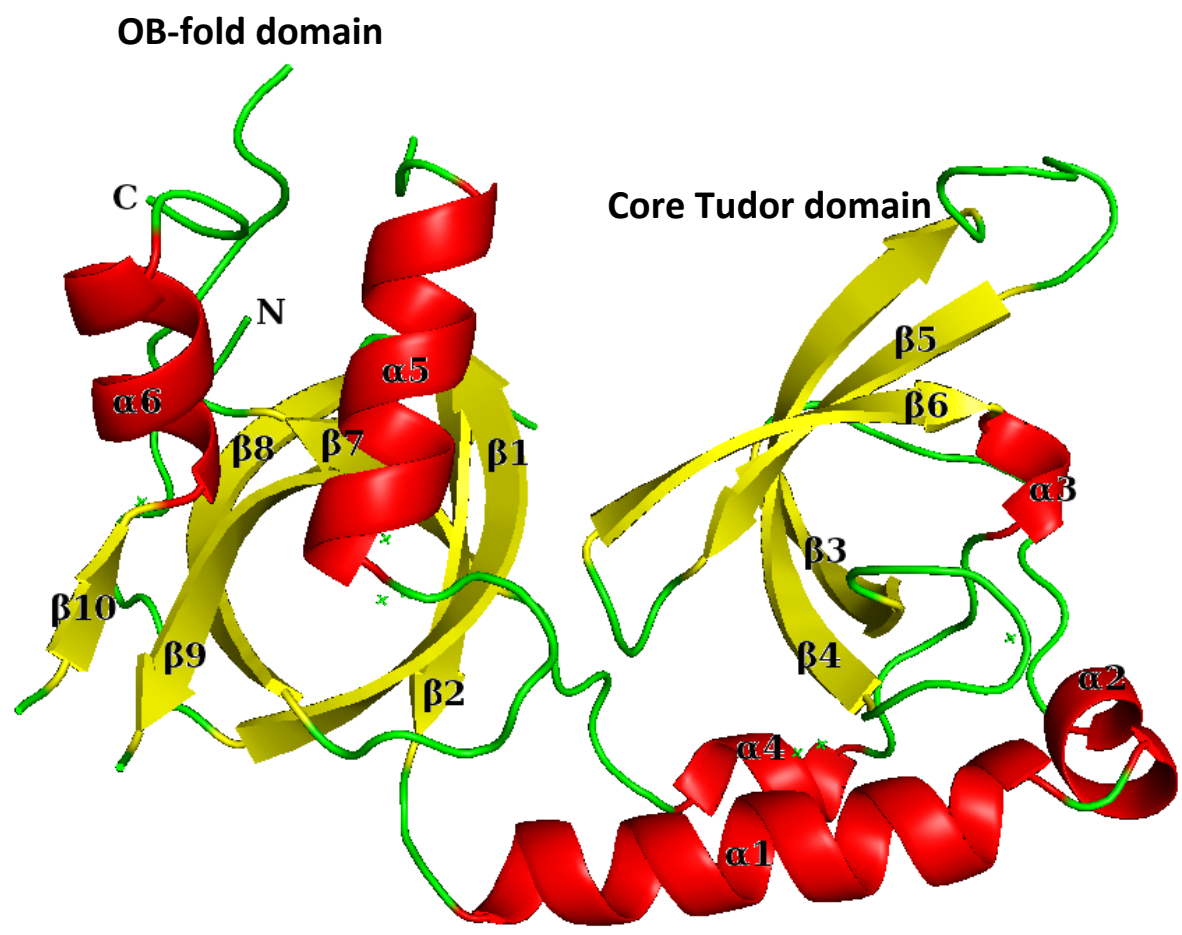

Figure 2. Crystal structure of BmPAPI extended Tudor domain from crystal form $\mathrm{P} 3_{2} 21$. $\alpha$ helices and $\beta$-sheets are labeled and shown in red and yellow respectively. Loops are shown in green. The OB-fold domain is comprised of $\beta 1-\beta 2, \beta 7-\beta 9$ and $\alpha 5$. The core Tudor domain consists of $\beta 3-\beta 6$. 
Figure 3
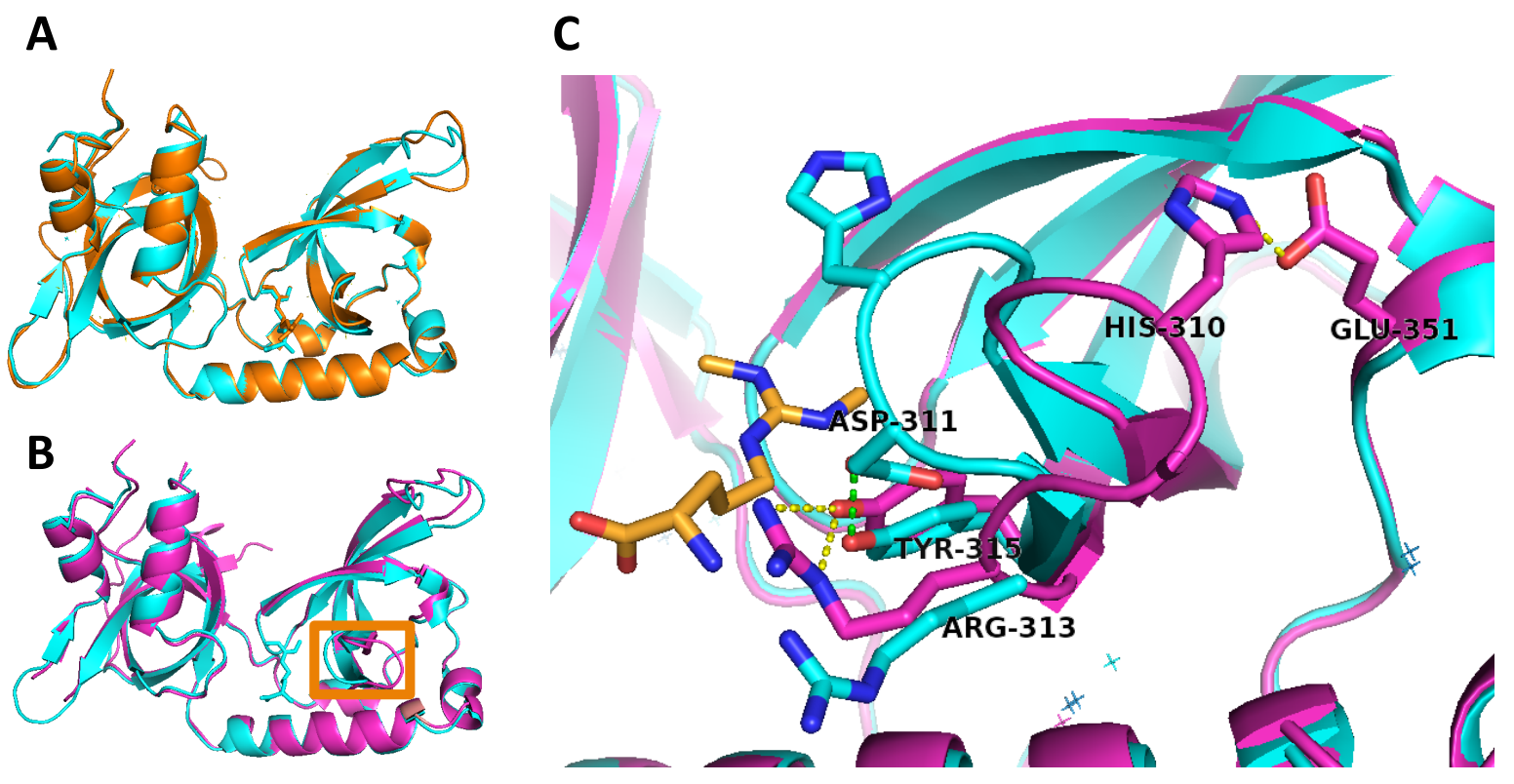

Figure 3. Crystal structure of the BmPAPI-sDMA complex. (A) Overlay of the two chains in the BmPAPI-sDMA structure. Chain $A$ is shown in orange and chain $B$ is shown in cyan. (B) Superposition of $\mathrm{P}_{2} 21$ apo-BmPAPI (magenta) and $\mathrm{P}_{2}{ }_{3}{ }_{1} 2$ sDMA-BmPAPI complex (chain $\mathrm{B}$, cyan) structures. A flexible loop that differs in conformation between the crystal forms is highlighted by the orange box. (C) A close-up view of the flexible loop region in native (magenta) and sDMA-bound (cyan) state. The sDMA is shown in orange. Blue and red indicate nitrogen and oxygen atoms respectively. Hydrogen bonds are shown as dashed lines (yellow for the native state and green for sDMA-bound state). 


\section{Figure 4}

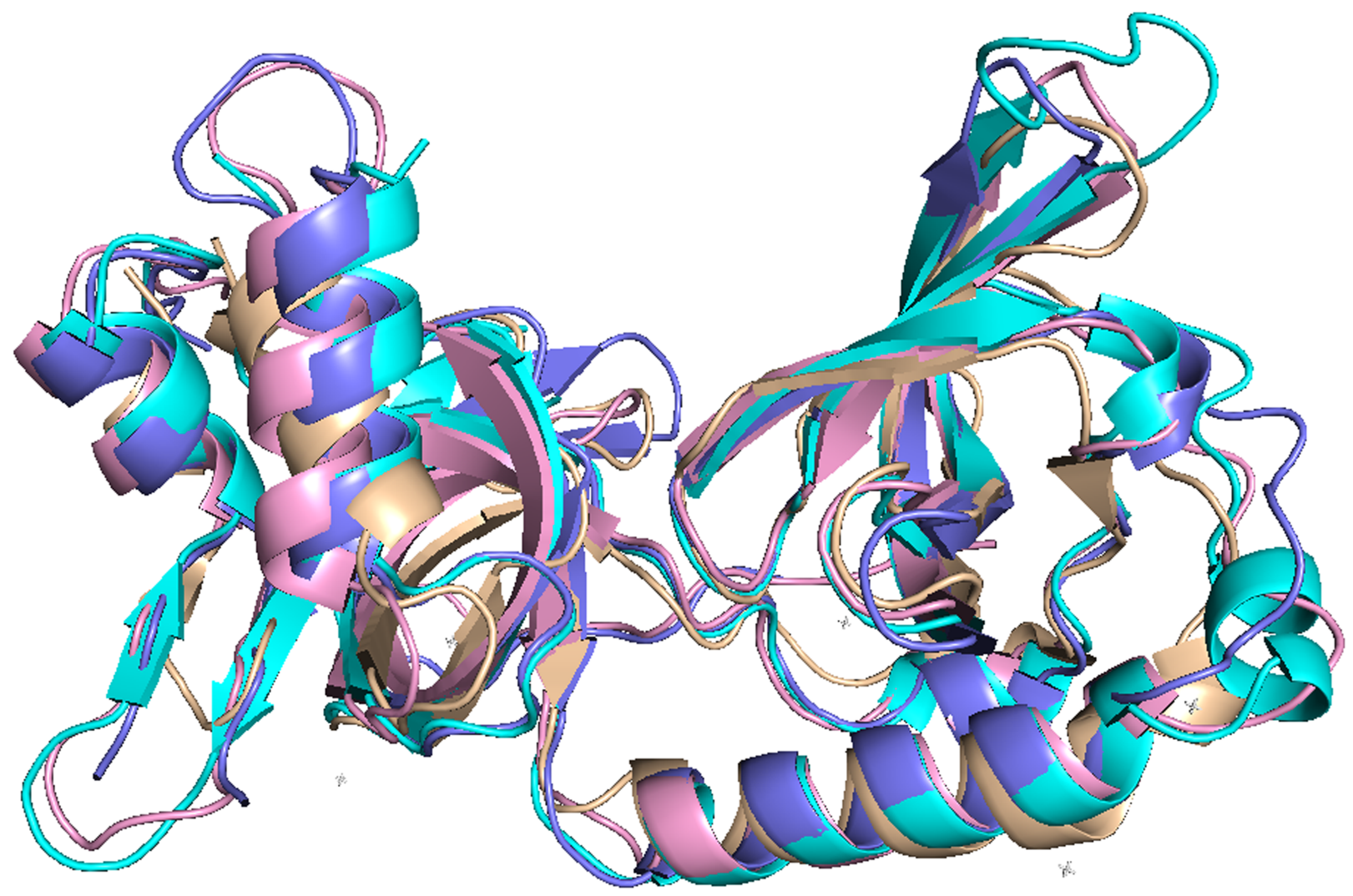

Figure 4. Superposition of the extended Tudor domain structures from BmPAPI homologs. Chain B of BmPAPI Tudor domain in the sDMA-bound state is shown in cyan; chain A of human Tdrd2 (PDB ID 5J39) is shown in lavender; chain A of Td3 domain from mouse Tdrd1 (PDB ID 4B9W) is shown in pink and eTud11 domain of Drosophila melanogaster Tudor protein (PDB ID $3 \mathrm{NTI})$ is shown in wheat color. 
Figure 5


Figure 5. sDMA binding of BmPAPI. (A) A zoomed-in view of the aromatic cage for sDMA binding. The sDMA and interacting residues are shown in sticks with the sDMA colored pink. Blue and red indicate nitrogen and oxygen atoms respectively. Hydrogen bonds are shown as yellow dashed lines, while purple dashed lines show the distances between the nitrogen atom in the coordinated amine group and the centers of aromatic rings. (B) Surface electron potential of the aromatic cage, generated with PyMOL (The PyMOL Molecular Graphics System, Version 1.8, Schrödinger, LLC). sDMA is modeled in the bound position and shown in green sticks. The color of the surface reflects a range of $-5.0 \mathrm{kT} / \mathrm{e}$ (red) to $+5.0 \mathrm{kT} / \mathrm{e}$ (blue), colored by potential on the solvent-accessible surface. (C, D) Front and back view of the surface electron potential of BmPAPI extended Tudor domain. The color of the surface reflects a range of $-3.0 \mathrm{kT} / \mathrm{e}$ (red) to $+3.0 \mathrm{kT} / \mathrm{e}$ (blue), colored by potential on the solvent accessible surface. 


\section{REFERENCES}

1. Cox DN, Chao A, Baker J, Chang L, Qiao D, Lin H. A novel class of evolutionarily conserved genes defined by piwi are essential for stem cell self-renewal. Genes \& development. 1998;12(23):3715-27.

2. Deng W, Lin H. miwi, a murine homolog of piwi, encodes a cytoplasmic protein essential for spermatogenesis. Developmental cell. 2002;2(6):819-30.

3. Kuramochi-Miyagawa S, Kimura T, ljiri TW, Isobe T, Asada N, Fujita Y, et al. Mili, a mammalian member of piwi family gene, is essential for spermatogenesis. Development (Cambridge, England). 2004;131(4):839-49.

4. Seto AG, Kingston RE, Lau NC. The coming of age for Piwi proteins. Molecular cell. 2007;26(5):603-9.

5. Malone CD, Hannon GJ. Molecular evolution of piRNA and transposon control pathways in Drosophila. Cold Spring Harbor symposia on quantitative biology. 2009;74:225-34.

6. Senti KA, Brennecke J. The piRNA pathway: a fly's perspective on the guardian of the genome. Trends in genetics : TIG. 2010;26(12):499-509.

7. Siomi MC, Sato K, Pezic D, Aravin AA. PIWI-interacting small RNAs: the vanguard of genome defence. Nature reviews Molecular cell biology. 2011;12(4):246-58.

8. Thomson $\mathrm{T}$, Lin $\mathrm{H}$. The biogenesis and function of PIWI proteins and piRNAs: progress and prospect. Annual review of cell and developmental biology. 2009;25:355-76.

9. Juliano $\mathrm{C}$, Wang J, Lin $\mathrm{H}$. Uniting germline and stem cells: the function of Piwi proteins and the piRNA pathway in diverse organisms. Annual review of genetics. 2011;45:447-69.

10. Hock J, Meister G. The Argonaute protein family. Genome biology. 2008;9(2):210.

11. Yan KS, Yan S, Farooq A, Han A, Zeng L, Zhou MM. Structure and conserved RNA binding of the PAZ domain. Nature. 2003;426(6965):468-74. 
12. Chen C, Jin J, James DA, Adams-Cioaba MA, Park JG, Guo Y, et al. Mouse Piwi interactome identifies binding mechanism of Tdrkh Tudor domain to arginine methylated Miwi. Proceedings of the National Academy of Sciences of the United States of America. 2009;106(48):20336-41.

13. Nishida KM, Okada TN, Kawamura T, Mituyama T, Kawamura Y, Inagaki S, et al. Functional involvement of Tudor and dPRMT5 in the piRNA processing pathway in Drosophila germlines. The EMBO journal. 2009;28(24):3820-31.

14. Vagin VV, Hannon GJ, Aravin AA. Arginine methylation as a molecular signature of the Piwi small RNA pathway. Cell cycle. 2009;8(24):4003-4.

15. Kirino Y, Kim N, de Planell-Saguer M, Khandros E, Chiorean S, Klein PS, et al. Arginine methylation of Piwi proteins catalysed by dPRMT5 is required for Ago3 and Aub stability. Nature cell biology. 2009;11(5):652-8.

16. Maurer-Stroh S, Dickens NJ, Hughes-Davies L, Kouzarides T, Eisenhaber F, Ponting CP. The Tudor domain 'Royal Family': Tudor, plant Agenet, Chromo, PWWP and MBT domains. Trends Biochem Sci. 2003;28(2):69-74.

17. Chen C, Nott TJ, Jin J, Pawson T. Deciphering arginine methylation: Tudor tells the tale. Nature reviews Molecular cell biology. 2011;12(10):629-42.

18. Siomi MC, Mannen T, Siomi H. How does the royal family of Tudor rule the PIWIinteracting RNA pathway? Genes \& development. 2010;24(7):636-46.

19. Vagin VV, Wohlschlegel J, Qu J, Jonsson Z, Huang X, Chuma S, et al. Proteomic analysis of murine Piwi proteins reveals a role for arginine methylation in specifying interaction with Tudor family members. Genes \& development. 2009;23(15):1749-62.

20. Mathioudakis N, Palencia A, Kadlec J, Round A, Tripsianes K, Sattler M, et al. The multiple Tudor domain-containing protein TDRD1 is a molecular scaffold for mouse Piwi proteins and piRNA biogenesis factors. RNA (New York, NY). 2012;18(11):2056-72. 
21. Pandey RR, Tokuzawa Y, Yang Z, Hayashi E, Ichisaka T, Kajita S, et al. Tudor domain containing 12 (TDRD12) is essential for secondary PIWI interacting RNA biogenesis in mice. Proceedings of the National Academy of Sciences of the United States of America. 2013;110(41):16492-7.

22. Liu K, Chen C, Guo Y, Lam R, Bian C, Xu C, et al. Structural basis for recognition of arginine methylated Piwi proteins by the extended Tudor domain. Proceedings of the National Academy of Sciences of the United States of America. 2010;107(43):18398-403.

23. Pek JW, Anand A, Kai T. Tudor domain proteins in development. Development (Cambridge, England). 2012;139(13):2255-66.

24. Lamb FS, Barna TJ, Goud C, Marenholz I, Mischke D, Schutte BC. Complex RNA processing of TDRKH, a novel gene encoding the putative RNA-binding tudor and $\mathrm{KH}$ domains. Gene. 2000;246(1-2):209-18.

25. Myrick LK, Hashimoto H, Cheng X, Warren ST. Human FMRP contains an integral tandem Agenet (Tudor) and $\mathrm{KH}$ motif in the amino terminal domain. Human molecular genetics. 2015;24(6):1733-40.

26. Rogne M, Landsverk HB, Van Eynde A, Beullens M, Bollen M, Collas P, et al. The KHTudor domain of a-kinase anchoring protein 149 mediates RNA-dependent self-association. Biochemistry. 2006;45(50):14980-9.

27. Liu L, Qi H, Wang J, Lin H. PAPI, a novel TUDOR-domain protein, complexes with AGO3, ME31B and TRAL in the nuage to silence transposition. Development (Cambridge, England). 2011;138(9):1863-73.

28. Honda S, Kirino Y, Maragkakis M, Alexiou P, Ohtaki A, Murali R, et al. Mitochondrial protein BmPAPI modulates the length of mature piRNAs. RNA (New York, NY). 2013;19(10):1405-18. 
29. Saxe JP, Chen M, Zhao H, Lin H. Tdrkh is essential for spermatogenesis and participates in primary piRNA biogenesis in the germline. The EMBO journal. 2013;32(13):186985.

30. Izumi N, Shoji K, Sakaguchi Y, Honda S, Kirino Y, Suzuki T, et al. Identification and Functional Analysis of the Pre-piRNA 3' Trimmer in Silkworms. Cell. 2016;164(5):962-73.

31. Murzin AG. OB(oligonucleotide/oligosaccharide binding)-fold: common structural and functional solution for non-homologous sequences. The EMBO journal. 1993;12(3):861-7.

32. Sprangers R, Groves MR, Sinning I, Sattler M. High-resolution X-ray and NMR Structures of the SMN Tudor Domain: Conformational Variation in the Binding Site for Symmetrically Dimethylated Arginine Residues. Journal of Molecular Biology. 2003;327(2):50720.

33. Altschul SF, Madden TL, Schaffer AA, Zhang J, Zhang Z, Miller W, et al. Gapped BLAST and PSI-BLAST: a new generation of protein database search programs. Nucleic acids research. 1997;25(17):3389-402.

34. Altschul SF, Wootton JC, Gertz EM, Agarwala R, Morgulis A, Schaffer AA, et al. Protein database searches using compositionally adjusted substitution matrices. The FEBS journal. 2005;272(20):5101-9.

35. Papadopoulos JS, Agarwala R. COBALT: constraint-based alignment tool for multiple protein sequences. Bioinformatics. 2007;23(9):1073-9.

36. Zhang H TW, Dong A, Bountra C, Arrowsmith CH, Edwards AM, Min J. Crystal structure of the extended Tudor domain from Tdrd2. J Structural Genomics Consortium.

37. Krissinel E, Henrick K. Secondary-structure matching (SSM), a new tool for fast protein structure alignment in three dimensions. Acta crystallographica Section D, Biological crystallography. 2004;60(Pt 12 Pt 1):2256-68.

38. Henrick EKaK. Multiple Alignment of Protein Structures in Three Dimensions. CompLife. 2005(LNBI 3695):67-78. 
39. Chuma S, Hiyoshi M, Yamamoto A, Hosokawa M, Takamune K, Nakatsuji N. Mouse Tudor Repeat-1 (MTR-1) is a novel component of chromatoid bodies/nuages in male germ cells and forms a complex with snRNPs. Mechanisms of development. 2003;120(9):979-90.

40. Reuter M, Chuma S, Tanaka T, Franz T, Stark A, Pillai RS. Loss of the Mili-interacting Tudor domain-containing protein-1 activates transposons and alters the Mili-associated small RNA profile. Nature structural \& molecular biology. 2009;16(6):639-46. 